\title{
GLI3 repressor controls functional development of the mouse ureter
}

\author{
Jason E. Cain, ${ }^{1,2}$ Epshita Islam, ${ }^{1}$ Fiona Haxho, ${ }^{1}$ Joshua Blake, ${ }^{1,3}$ and Norman D. Rosenblum ${ }^{1,2,3,4,5}$ \\ ${ }^{1}$ Program in Developmental and Stem Cell Biology and ${ }^{2}$ Division of Nephrology, Hospital for Sick Children, \\ Toronto, Ontario, Canada. ${ }^{3}$ Department of Physiology, ${ }^{4}$ Department of Pediatrics, and \\ ${ }^{5}$ Department of Laboratory Medicine and Pathobiology, University of Toronto, Toronto, Ontario, Canada.
}

\begin{abstract}
Obstructive and nonobstructive forms of hydronephrosis (increased diameter of the renal pelvis and calyces) and hydroureter (dilatation of the ureter) are the most frequently detected antenatal abnormalities, yet the underlying molecular mechanisms are largely undefined. Hedgehog $(\mathrm{Hh})$ proteins control tissue patterning and cell differentiation by promoting GLI-dependent transcriptional activation and by inhibiting the processing of GLI3 to a transcriptional repressor. Genetic mutations that generate a truncated GLI3 protein similar in size to the repressor in humans with Pallister-Hall syndrome (PHS; a disorder whose characteristics include renal abnormalities) and hydroureter implicate $\mathrm{Hh}$-dependent signaling in ureter morphogenesis and function. Here, we determined that Hh signaling controls 2 cell populations required for the initiation and transmission of coordinated ureter contractions. Tissue-specific inactivation of the Hh cell surface effector Smoothened (Smo) in the renal pelvic and upper ureteric mesenchyme resulted in nonobstructive hydronephrosis and hydroureter characterized by ureter dyskinesia. Mutant mice had reduced expression of markers of cell populations implicated in the coordination of unidirectional ureter peristalsis (specifically, Kit and hyperpolarization-activation cation-3 channel [Hcn3]), but exhibited normal epithelial and smooth muscle cell differentiation. Kit deficiency in a mouse model of PHS suggested a pathogenic role for GLI3 repressor in Smo-deficient embryos; indeed, genetic inactivation of Gli3 in Smo-deficient mice rescued their hydronephrosis, hydroureter, Kit and $\mathrm{Hcn} 3$ expression, and ureter peristalsis. Together, these data demonstrate that $\mathrm{Hh}$ signaling controls Kit and Hon 3 expression and ureter peristalsis.
\end{abstract}

\section{Introduction}

Congenital dilatation of the urinary tract, defined as increased diameter of the renal pelvis (hydronephrosis) with or without dilatation of the ureter (hydroureter), is the most frequently detected abnormality by prenatal ultrasound, with an incidence of $0.5 \%-1 \%(1)$. Although antenatal urinary dilation is frequently transient, approximately $20 \%-30 \%$ of cases fail to resolve spontaneously and become clinically significant (2). Indeed, congenital obstructive nephropathy is a common cause of renal failure in infants and children (3). Approximately $50 \%$ of all cases of urinary tract dilatation have no evidence of a physical obstruction and no abnormality in the structural components of the pelvic or ureteral wall. Despite the assumption of a functional obstruction in such cases, the underlying pathogenic mechanisms that contribute to impaired ureter peristalsis are unknown.

The Hedgehog-GLI (Hh-GLI) signaling pathway plays a critical role in kidney and ureter morphogenesis (4-8). In vertebrates, the members of the GLI family of proteins - GLI1, GLI2, and GLI3 mediate Hh signaling. Hh ligand binds its transmembrane receptor, Patched1 (PTCH1), relieving inhibition of Smoothened (SMO) and triggering SMO localization to the cilium. In this state, full-length GLI activators translocate to the nucleus and activate gene transcription. In the absence of Hh ligand, PTCH1 inhibits the ciliary localization of SMO and prevents its interaction with an intracellular molecular complex consisting of KIF7/KIF27, Fused (FU), and Suppressor of Fused (SUFU). In this state, the KIF7/KIF27-FU-SUFU complex is able to bind full-length GLI proteins, ultimately resulting in C-terminal cleavage of GLI3, which translocates to the nucle-

Conflict of interest: The authors have declared that no conflict of interest exists. Citation for this article: JClin Invest. 2011;121(3):1199-1206. doi:10.1172/JCI45523. us and represses gene transcription (9). The response to Hh ligand in any particular cell is dependent on the intracellular balance of GLI activators and GLI3 repressor. Shb deficiency targeted to the ureteric cell lineage results in decreased ureter mesenchyme proliferation, delayed ureter smooth muscle differentiation, and severe hydroureter, suggestive of a critical role for Hh signaling in ureter morphogenesis (8). Humans and mice with mutations predicted to generate a truncated protein similar in size to GLI3 repressor exhibit hydroureter and hydronephrosis, although the underlying mechanisms in these cases are undefined $(2,10)$.

Although smooth muscle is required for ureter peristalsis, the mechanisms that control peristaltic activity are unknown. Interstitial cells of Cajal-like cells (ICC-LCs), marked by Kit expression, are localized throughout the upper urinary tract and have morphologic and molecular characteristics similar to those of intestinal pacemaker cells (11-13). Inhibition of Kit activity in ureter explants using neutralizing antibodies results in perturbation of unidirectional peristalsis, but not smooth muscle cell differentiation, which suggests that Kit function is required for ureter peristalsis (11). More recently, a cell population residing in the pelvis-kidney junction that expresses hyperpolarization-activated cation-3 channel (Hcn3) has been described (14). Hyperpolarization-activated cation $(\mathrm{HCN})$ channels are implicated in spontaneous membrane polarizations of heart pacemaker cells $(15,16)$. Inhibition of $\mathrm{HCN}$ ion channel activity in urinary tract explants results in loss of coordinated, proximal-distal ureter contractions, which suggests a requirement for $H c n 3$ function in ureter peristalsis (14). Yet the molecular mechanisms regulating Kit and Hcn3 are unknown.

Here, we have identified what we believe to be a novel function of the Hh signaling pathway in the establishment and maintenance of coordinated unidirectional proximal-distal ureter contractions via 
control of Kit and Hcn3 expression. We propose a model whereby Hh signaling activity, and thereby absence of GLI3 repressor function, has a multifunctional role in the functional development of the ureter, being (a) required for normal ureter smooth muscle cell development and differentiation and (b) necessary for the control of Kit and Hcn 3 expression in 2 distinct cell populations required for initiation and transmission of ureter peristalsis.

\section{Results}

Smo deficiency causes congenital bydroureter and bydronephrosis. Hh signaling activity, reported by Ptch1-lacZ expression, within ureteric mesenchyme during embryogenesis suggests a functional role for Hh signaling during kidney-urinary development (4). Yet embryonic lethality in Smo-deficient embryos prior to the onset of urinary tract development has limited elucidation of Hh signaling functions (17). Therefore, we generated mice in which Smo inactivation is restricted to the intermediate mesoderm (Rarb2-Cre; $S m o^{l o x P /-}$ mice) using $S m o^{l o x P}$ mice and Rarb2-Cre mice, in which expression of Cre recombinase is controlled by a Rarb2 promoter (18). Analysis of the temporal and spatial activity of Rarb2-Cre was analyzed using the ROSA reporter mouse strain. In Rarb2-Cre;ROSA mice at E11.5, a stage at which the ureteric bud has branched once within the metanephric mesenchyme, lac $Z$ activity was localized to the metanephric mesenchyme and absent from the periureteric mesenchyme (Figure 1, A and B, and ref. 18). Beginning at E15.5, lac Z activity was observed in the periureteric mesenchyme in addition to the metanephric mesenchyme (Figure 1, C-F). Taken together, these data demonstrate that CRE-mediated deletion in the periureteric mesenchyme occurs after the onset of kidney development in Rarb2-Cre;Smo loxP/- mice.

Rarb2-Cre;Smo ${ }^{\text {loxP/- }}$ mice were recovered at birth in the numbers predicted by Mendelian segregation, indicating no adverse effect of Smo deficiency on embryonic viability. However, many mutants failed to thrive in the postnatal period and died within the first 2 months of life. Smo deletion was confirmed using quantitative real-time PCR on E11.5 metanephric mesenchyme dissected free of ureteric branches. Smo mRNA transcripts were reduced by approximately $65 \%$ in metanephric mesenchyme isolated from Rarb2-Cre;Smo ${ }^{\text {loxP/- }}$ mice (control, $0.096 \pm 0.015$; Rarb2-Cre;Smo ${ }^{\text {loxP } /-}$, $0.036 \pm 0.009 ; P<0.05)$. The lack of absolute Smo mRNA deficiency in mutant mice is likely explained by the heterogeneity of Rarb2-Cre expression in the metanephric mesenchyme (18). E18.5 Rarb2-Cre; $S m o^{l o x P /-}$ mice demonstrated bilateral hydroureter and hydropelvis (16 of $22 ; 72 \%$ ), characterized by dilation of the proximal ureter and renal pelvis, and ablation of the renal papilla and medullary tissue. In contrast, bilateral hydroureter was never observed in mice heterozygous for Smo deficiency (Smo ${ }^{l o x P} /-$ and Rarb2-Cre; $\left.S m o^{l o x P /+}\right)$, although mild unilateral hydroureter was observed in $17 \%$ of these mice ( 7 of 42 ; Figure 1, G-J). Urinary tract dilatation was never observed in WT or $S m o^{l o x P /+}$ control mice ( 0 of 18). To determine whether a physical obstruction contributes to hydroureter and hydronephrosis in Rarb2-Cre;Smo ${ }^{\text {loxP } /-}$ embryos, we performed intrapelvic dye injection in E18.5 mouse embryos. Similar to control mice, dye was readily cleared from Rarb2-Cre; Smo ${ }^{\text {loxP } /-}$ kidneys and pooled in the bladder (Figure 1, K and L). These data indicate that Rarb2-Cre-mediated Smo deletion causes nonobstructive hydronephrosis and hydroureter.

Mechanisms that determine the structural integrity of the renal pelvis and ureter have been previously defined. Here we investigated the relevance of these mechanisms in Rarb2-Cre;Smo ${ }^{\text {loxP/- }}$ mice.
Mutations in genes that control urothelial and smooth muscle cell differentiation result in nonobstructive hydroureter and hydronephrosis $(8,19-24)$. Histologic analysis of the ureter in Rarb2-Cre; $S m o^{l o x P} /-$ mice, including those with mild or severe hydronephrosis, demonstrated normal urothelial, subepithelial, smooth muscle, and outer connective tissue layers. Moreover, analysis of urothelial differentiation, via uroplakin III (UpkIII) expression, and ureteral smooth muscle differentiation, via $\alpha$-SMA expression, revealed normal cell differentiation in these mice (Figure 1, $\mathrm{M}-\mathrm{R})$. Hydronephrosis can also arise from a delay, but not an absolute deficiency, in ureteral smooth muscle cell differentiation (8). Thus, we analyzed the ontogeny of ureteric smooth muscle differentiation in Rarb2-Cre;SmoloxP/- mice using immunofluorescence microscopy and did not observe any delay in smooth muscle differentiation (Supplemental Figure 1; supplemental material available online with this article; doi:10.1172/JCI45523DS1).This is likely because the onset of Rarb2-Cre expression in the ureter mesenchyme occurs after the critical period of smooth muscle cell development. Abnormal ureteric branching has been implicated in medullary malformation and subsequent hydropelvis and hydroureter (25-27). Analysis of Rarb2-Cre;Smo ${ }^{\text {loxP/- }}$ and control $S m o^{l o x P /+}$ mice at E12.5 revealed comparable branch number (control, $65.6 \pm 15.5$ branches, $n=13$; Rarb2-Cre;Smo ${ }^{\text {loxP } /-}, 52.6 \pm 22.3$ branches, $n=10 ; P=0.113$ ) and pattern as well as comparable expression of Ret and Wnt11, which are functionally required in ureter tip cells for branching morphogenesis (Supplemental Figure 2). Furthermore, histological analysis of medullary formation from E15.5 to E18.5, and expression of medullary collecting duct markers $W n t 7 b$ and $W n t 9 b$, were indistinguishable from control littermates (Supplemental Figure 3). Taken together, these data indicate that hydronephrosis and hydroureter in Rarb2-Cre;SmoloxP/- mice is not associated with abnormal collecting duct formation or structural maldevelopment of the pelvis and upper ureter.

Smo-deficient embryos exhibit functional ureter obstruction. We investigated the contribution of abnormal ureter function to hydronephrosis and hydroureter in Rarb2-Cre;Smo ${ }^{l o x P /-}$ mice by analyzing ureter peristalsis using an in vitro culture system. In control ureters, peristalsis occurred in a fluent wave-like motion, commencing in the renal pelvis/proximal ureter and moving distally (Figure 1, S and U, and Supplemental Video 1). In contrast, we observed severe dyskinesia in ureters isolated from Rarb2-Cre; SmoloxP/- mice (Figure 1, T and V, and Supplemental Video 2). Although Rarb2-Cre; SmoloxP/- ureters demonstrated strong contractions, peristalsis failed to propagate in a sequential proximal-distal direction. Rather, we observed ectopic initiation of the contraction, failure of the contraction to propagate distally, and, in some cases, the complete absence of segmental contraction. These data suggest that hydroureter and hydronephrosis in Rarb2-Cre;SmoloxP/- mice result from a functional obstruction caused by abnormal ureter peristalsis.

Our finding of dysregulated ureter contractions in Rarb2-Cre; $S m o^{l o x P /-}$ ureters prompted us to examine mechanisms by which peristalsis is initiated and how the unidirectional coordination of the contraction is maintained and propagated. ICC-LCs are considered to act as urinary electrical pacemaker cells, instigating smooth muscle contraction (12). ICC-LCs are defined by their characteristic spindle shape and immunoreactivity to Kit in the mature upper urinary tract of mice and humans $(28,29)$. Immunofluorescence analysis demonstrated spindle-shaped, Kitpositive cells characteristic of ICC-LCs located in close proximity 

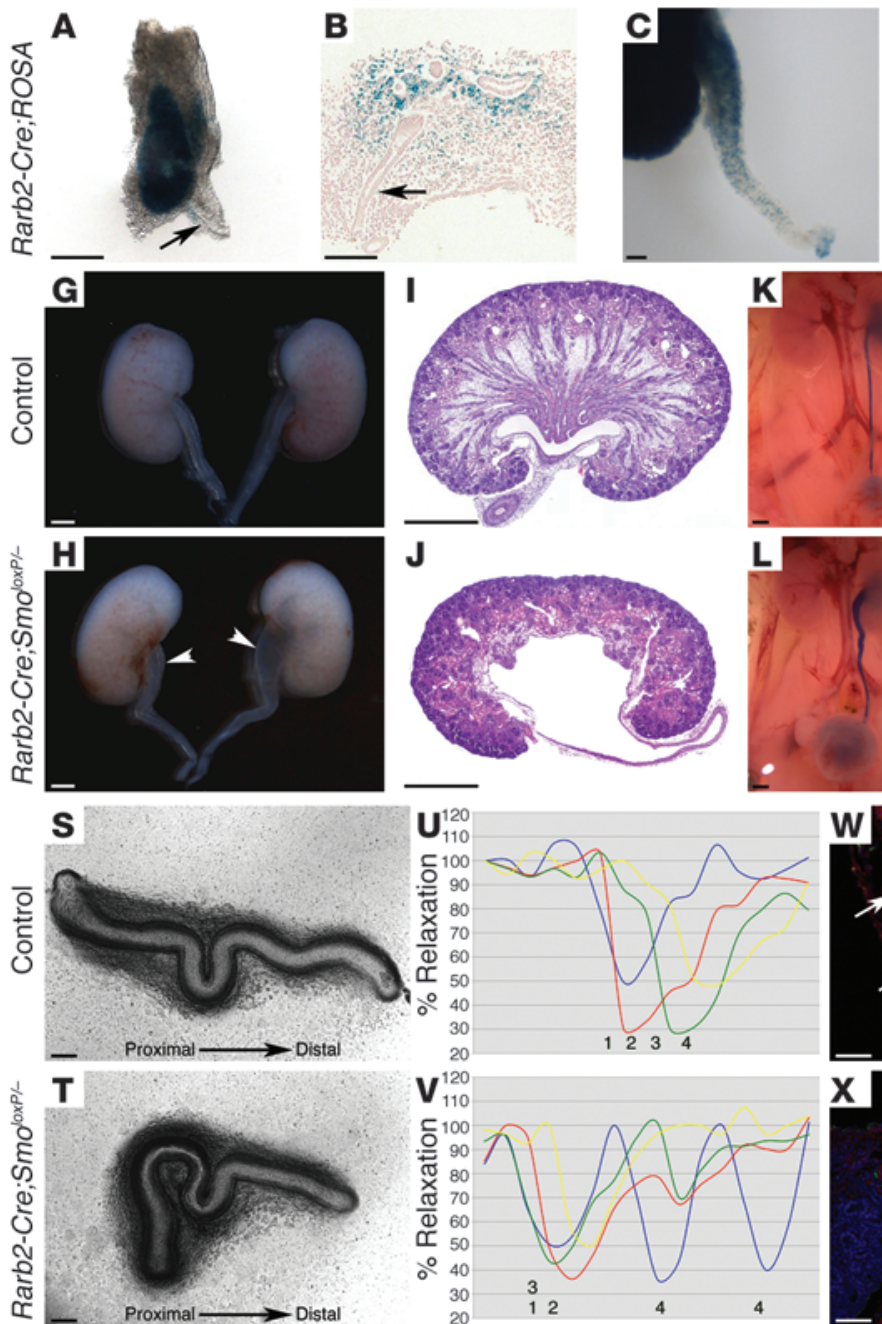

$\mathbf{J}$
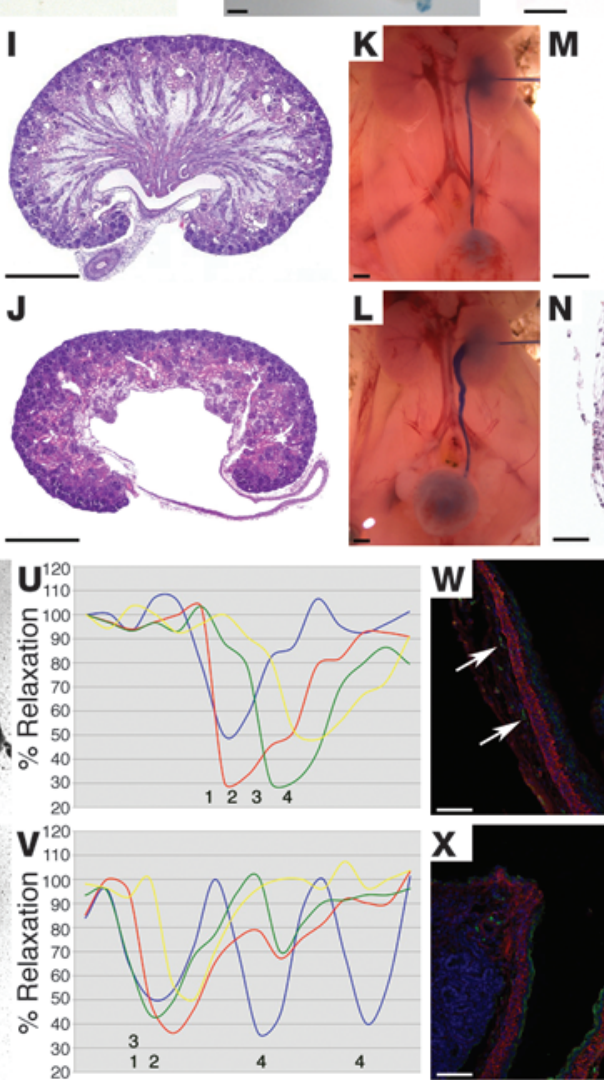

D
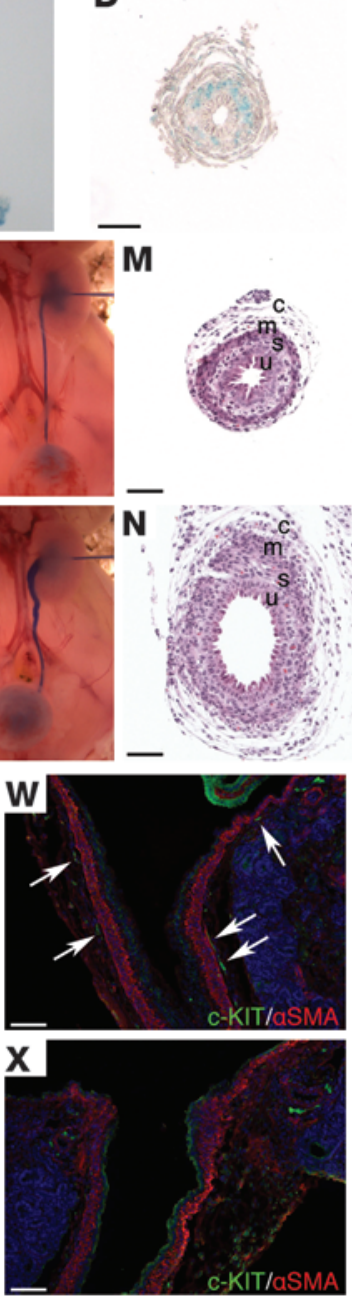
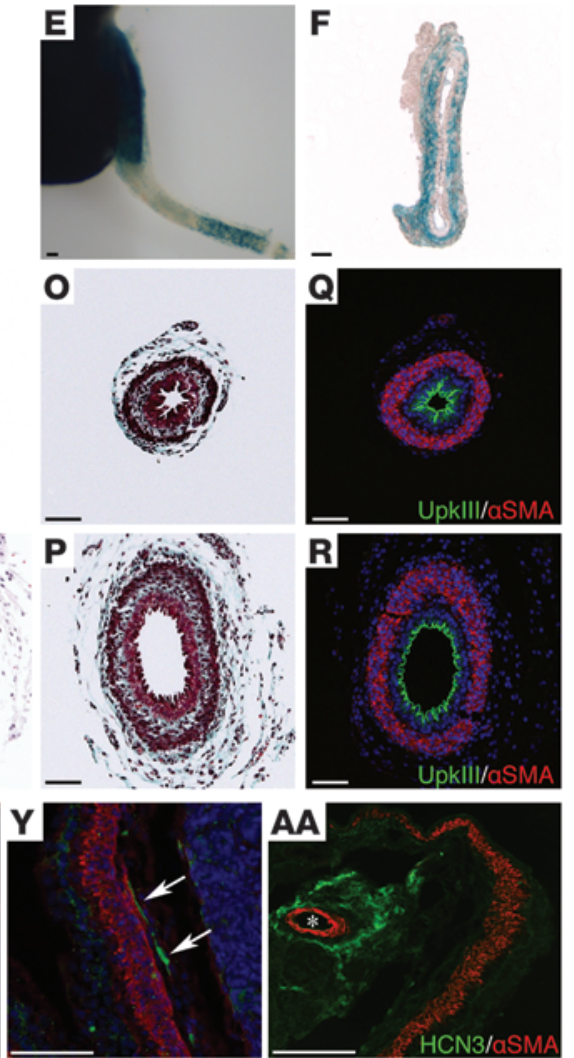

AA
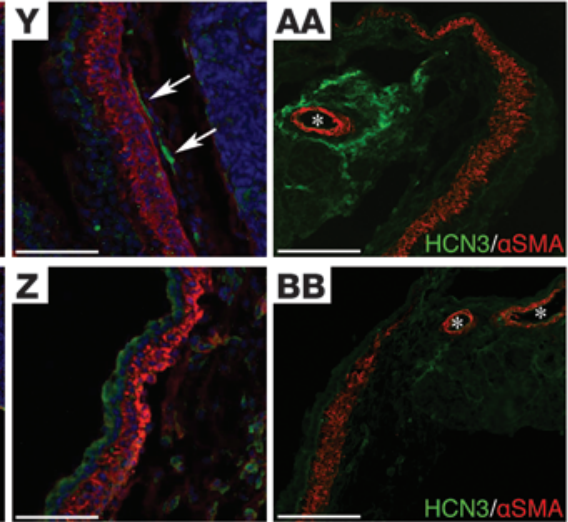

Figure 1

Smo deficiency targeted to the ureter mesenchyme results in nonobstructive hydroureter and hydronephrosis. (A-F) Rarb2-Cre activity was notably absent from the cells surrounding the ureteric bud (arrow) at E11.5 (A and B), was weakly localized to the developing smooth muscle layer at E15.5 (C and D), and was strongly expressed in all mesenchyme-derived ureter cell layers of the proximal ureter at E18.5 (E and F). (G-J) E18.5 Rarb2-Cre;SmoloxP/- mutants exhibited hydroureter and hydronephrosis. Arrowheads in $\mathbf{H}$ denote dilated proximal ureter. (K and $\mathbf{L}$ ) Nonobstructive hydroureter of Rarb2-Cre;SmoloxPl- mice, as demonstrated by intrapelvic dye injection. (M-R) Smo-deficient mutants did not exhibit ureter structural abnormalities, as indicated by hematoxylin and eosin staining ( $\mathbf{M}$ and $\mathbf{N})$, Masson trichrome stain for collagen-rich connective tissue layers ( $\mathbf{O}$ and $\mathbf{P})$, and normal expression of smooth muscle and specialized apical urothelium markers $\alpha$-SMA and UpkIII, respectively $(\mathbf{Q}$ and $\mathbf{R})$. u, urothelium; s, subepithelial stroma layer; $\mathrm{m}$, smooth muscle layer; c, outer connective tissue layer. (S-V) Analysis of ureter peristalsis in isolated ureters revealed an absence of coordinated ureter contractions in the Rarb2-Cre;SmoloxP/- mutant (blue line [1], proximal ureter; red line [2], midproximal ureter; green line [3], mid-distal ureter; yellow line [4], distal ureter). In $\mathbf{U}$ and $\mathbf{V}$, percent relaxation is plotted for

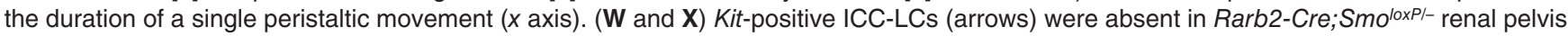
and proximal ureters. (Y and $\mathbf{Z}$ ) Higher-magnification views of $\mathbf{W}$ and $\mathbf{X}$. (AA and $\mathbf{B B}$ ) Hcn3, a marker of a pelvic-kidney cell population required for ureter contraction initiation, was markedly reduced in Rarb2-Cre;SmoloxP/- mutants. Asterisks denote renal arterioles. Scale bars: 100 um (A-F, M-T, and W-BB), $500 \mu \mathrm{m}(\mathbf{G}-\mathbf{L})$.

to the smooth muscle layer of the renal pelvis and proximal ureter in the E18.5 urinary tract of controls (Figure 1, W and Y). In contrast, Kit-positive ICC-LCs were undetectable in the renal pelvis or proximal ureter of Rarb2-Cre;SmoloxP/- mice (Figure 1, X and Z).

Ureter contractions are initiated in the pelvis-kidney junction and are considered critical for the coordination of downstream contractile activity. Recently, spontaneous membrane polarizations required for the initiation of ureter peristalsis in this region have been demonstrated to be under the control of
Hon3 (14). To determine whether abnormal initiation of peristalsis in the pelvis-kidney junction might also contribute to functional obstruction in Rarb2-Cre;SmoloxP/- embryos, we analyzed the expression of Hcn3. In control mice, Hcn3 was localized to a specific region of connective tissue within the renal pelvis (Figure 1AA and ref. 14). Remarkably, Hcn3 expression was substantially reduced in the renal pelvis of Rarb2-Cre;SmoloxP/embryos (Figure 1BB). Taken together, these reductions in Kit and Hcn 3 suggest that abnormal peristaltic initiation and trans- 

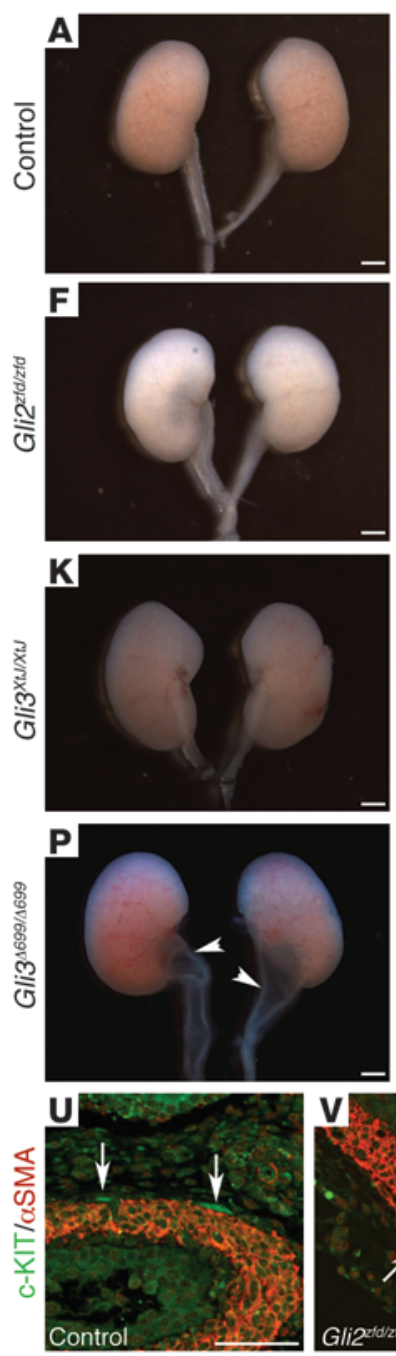

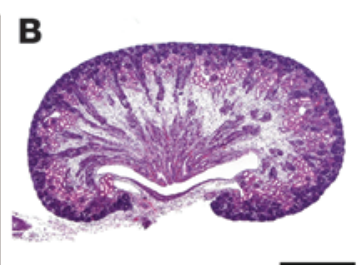

G

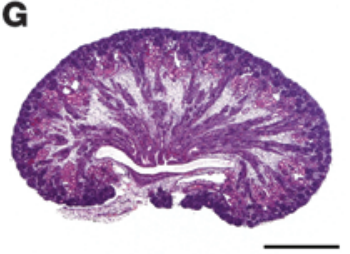

$\mathbf{L}$

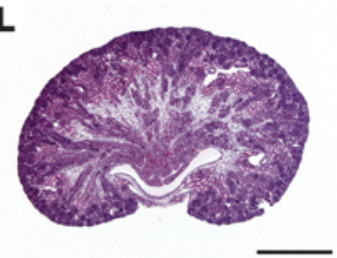

$\mathbf{Q}$

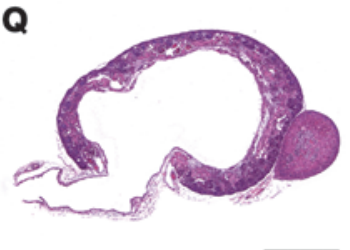

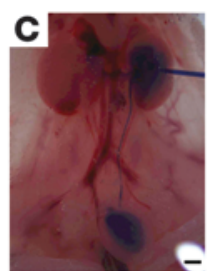
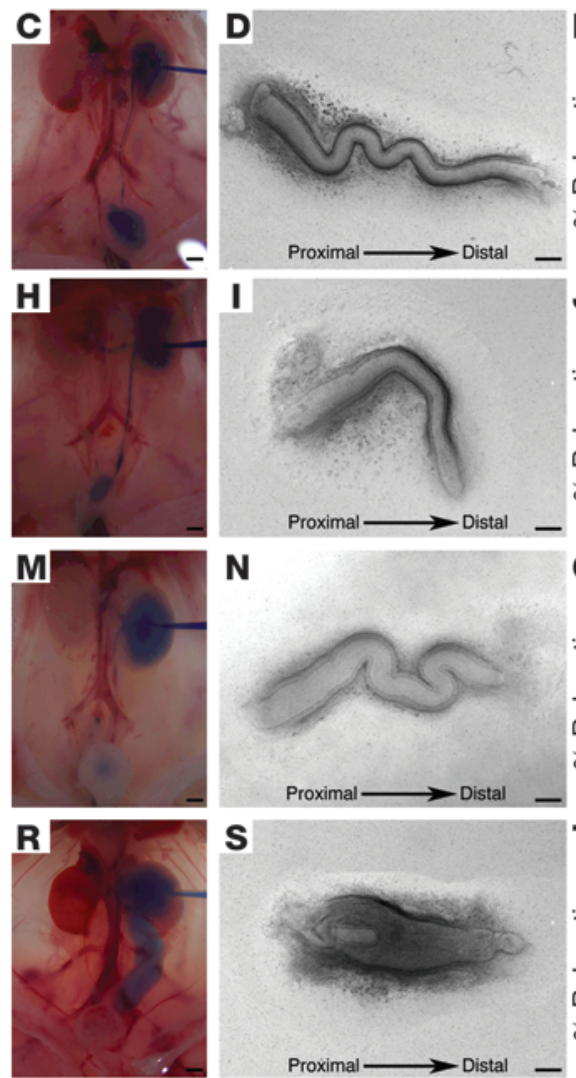

S
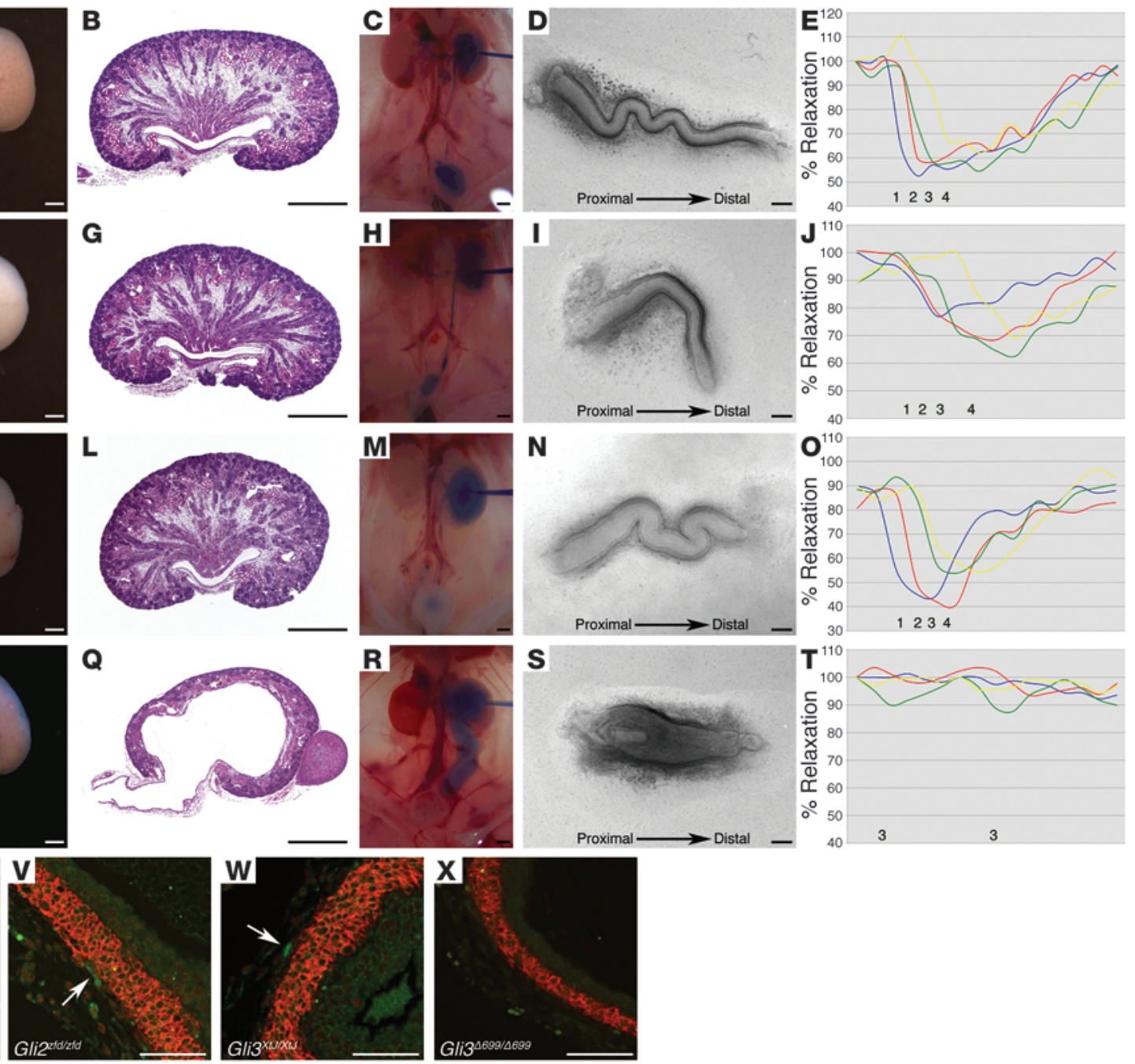

Figure 2

GLI3 repressor is deleterious to the functional development of the ureter. (A-T) E18.5 Gli2 homozygous mutants and Gli3 homozygous mutants exhibited normal kidney and ureter morphology $(\mathbf{F}, \mathbf{G}, \mathbf{K}$, and $\mathbf{L})$, normal ureter lumen diameter and dye clearance $(\mathbf{H}$ and $\mathbf{M})$, and coordinated proximal-distal ureter contractions (I, J, N, and $\mathbf{O}$; blue line [1], proximal ureter; red line [2], midproximal ureter; green line [3], mid-distal ureter; yellow line [4], distal ureter) compared with those of control littermates (A-E). The Gli3 ${ }^{\Delta 699 / \Delta 699}$ mouse model demonstrated severe nonobstructive hydroureter and hydronephrosis $(\mathbf{P}-\mathbf{R})$ and ureter dyskinesia $(\mathbf{S}$ and $\mathbf{T})$. In $\mathbf{E}, \mathbf{J}, \mathbf{O}$, and $\mathbf{T}$, percent relaxation is plotted for the duration of a single peristaltic movement ( $x$ axis). (U-X) Smooth muscle differentiation and Kit-positive ICC-LCs (arrows) were comparable among control (U), Gli2zfd/zfd $(\mathbf{V})$, and Gli3 Xt/IXtJ $(\mathbf{W})$ proximal ureters. In contrast, despite the presence of smooth muscle, Kit-positive ICC-LCs were undetectable in Gli3 ${ }^{\Delta 699 / \Delta 699}$ proximal ureters $(\mathbf{X})$. Scale bars: $100 \mu \mathrm{m}(\mathbf{D}, \mathbf{I}, \mathbf{N}, \mathbf{S}$, and $\mathbf{U}-\mathbf{X}), 500 \mu \mathrm{m}(\mathbf{A}-\mathbf{C}, \mathbf{F}-\mathbf{H}, \mathbf{K}-\mathbf{M}$, and $\mathbf{P}-\mathbf{R})$.

mission are the underlying mechanisms causing ureter dyskinesia, functional obstruction, and hydroureter and hydronephrosis in Rarb2-Cre;Smo ${ }^{l o x P /-}$ mice.

Elevated levels of GLI3 repressor are deleterious to the functional development of the ureter. Hh signaling increases activity of GLI transcriptional activators and inhibits formation of GLI3 transcriptional repressor. GLI2 is the primary GLI activator in mammals (9). During urinary development, homozygous Shb deficiency or inhibition of SMO by the pharmacological inhibitor cyclopamine, decreases levels of GLI activator proteins and reverses the ratio of GLI activators to GLI3 repressor in favor of GLI3 repressor (6). We investigated the functional contribution of GLI2 activator and GLI3 repressor to the functional development of the ureter by analyzing ureter morphogenesis and function in Gli2 and Gli3 homozygous mutant mice. Whole-mount and histological analysis of E18.5 Gli2-null urinary systems revealed no observable differences from control littermates (Figure 2, A, B, F, and $G$ ). Intrapelvic dye injections and analyses of ureter peristalsis also demonstrated normal ureter lumen diameter and sequential proximal-distal coordination of ureter contraction in Gli2-null mice (Figure 2, C-E and H-J, and Supplemental Videos 3 and 4). Similarly, no morphological or functional abnormalities in ureter morphogenesis were observed in E18.5 Gli3-null urinary systems (Figure 2, K-O, and Supplemental Video 5). Together, these results suggest that neither the major GLI activator (GLI2) nor GLI3 repressor is required for normal functional development of the ureter.

Next we investigated the contribution of increased levels of GLI3 repressor to hydroureter and hydronephrosis in a murine model of Pallister-Hall syndrome (PHS) in which a targeted mutation gen- 

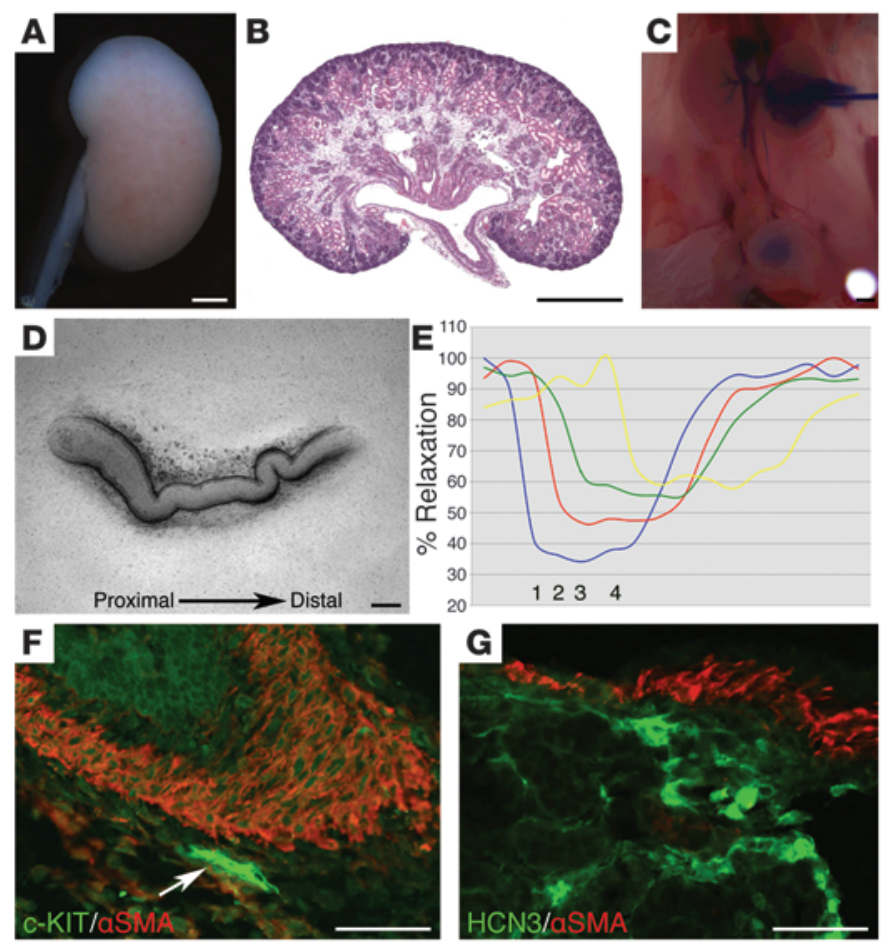

H

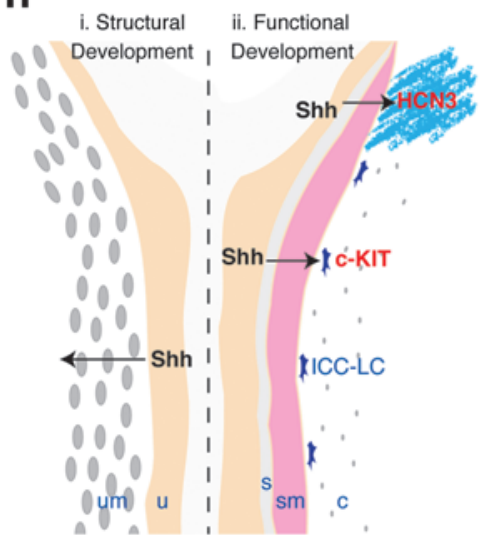

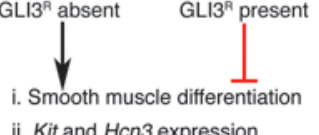

ii. Kit and $\mathrm{Hcn} 3$ expression

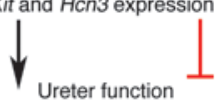

Figure 3

Elimination of GLI3 repressor in the Smo-deficient background rescues the renal phenotype. (A-G) Analysis of Rarb2-Cre;SmoloxPl-;Gli3XtJ/XtJ embryos at E18.5 revealed normalization of kidney and ureter morphology $(\mathbf{A}-\mathbf{C})$, rescue of coordinated proximal-distal ureter contractions (D and E; blue line [1], proximal ureter; red line [2], midproximal ureter; green line [3], mid-distal ureter; yellow line [4], distal ureter), and restoration of Kit $(\mathbf{F})$ and $H c n 3(\mathbf{G})$ expression. In E, percent relaxation is plotted for the duration of a single peristaltic movement ( $x$ axis). Scale bars: $100 \mu \mathrm{m}(\mathbf{D}, \mathbf{F}$, and $\mathbf{G}), 500 \mu \mathrm{m}(\mathbf{A}-\mathbf{C})$. (H) Model for a bifunctional role for Hh signaling during the functional development of the ureter. (i) Shh secreted by the ureteric bud/urothelium is required during early development for smooth muscle cell development and the patterning of the ureter mesenchyme. (ii) During later development, SHH-SMO dependent signaling is required for Kit and Hcn3 expression in distinct cell populations that regulate coordinated ureter contractions. SHH signaling to adjacent cells promotes pathway activation and prevents formation of GLI3 repressor $\left(\mathrm{GLI} \mathrm{3}^{\mathrm{R}}\right)$. In the presence of GLI3 repressor function during ureter morphogenesis, smooth muscle cell differentiation and expression of Kit and Hcn3 is impaired, leading to abnormal ureter function. u, ureteric bud/urothelium; um, ureter mesenchyme; s, subepithelial layer; sm, smooth muscle; c, outer connective tissue.

erates a 699-amino acid N-terminal GLI3 protein similar to GLI3 repressor (10). Whole-mount and histological analyses of E18.5 Gli3 ${ }^{\Delta 699}$ homozygous urinary systems demonstrated severe hydroureter and hydropelvis (Figure 2, P and Q). Intrapelvic dye injection revealed a severely dilated but patent ureter lumen, thus excluding physical obstruction as a cause of urinary system dilatation (Figure 2R). Analysis of ureter peristalsis in Gli3 ${ }^{\Delta 699}$ homozygous isolated ureters demonstrated a loss of coordinated, proximal-distal contractions and weak spontaneous contractions of random ureter segments (Figure 2, S and T, and Supplemental Videos 6 and 7). Since we observed reduced Kit expression in the renal pelvis and proximal ureter of Rarb2-Cre;Smo ${ }^{\text {loxP/- }}$ embryos, we analyzed Kit expression in Gli3 ${ }^{\Delta 699}$ homozygous mutants. Consistent with normal morphology and function in Gli2 and Gli3 homozygous ureters, we observed no abnormalities in the smooth muscle layer or Kit-positive ICC-LCs in the renal pelvis and proximal ureter (Figure 2, U-W). In contrast, despite the presence of a normal smooth muscle layer, Kit-positive ICC-LCs were undetectable in the renal pelvis and proximal ureter of E18.5 Gli3 ${ }^{\Delta 699}$ homozygous embryos (Figure 2X). The strikingly similar morphological and functional phenotypes between Rarb2-Cre;Smo ${ }^{\text {loxP/-}}$ and Gli3 ${ }^{\Delta 699}$ homozygous ureters strongly suggest that GLI3 repressor is deleterious to the functional development of the ureter. To further investigate the functional contribution of GLI3 repressor to ureter function, we generated embryos with both Smo deficiency targeted to the ureter mesenchyme and Gli3 deficiency. Remarkably, elimination of Gli3 in the Rarb2-Cre; Smo ${ }^{\text {loxP/- }}$ background completely rescued hydroureter and hydronephrosis ( 0 of 7 Rarb2-Cre;Smo ${ }^{\text {loxP/-; }}$ Gli3 ${ }^{X t / X t J}$ mice; Figure 3, A-C) and normalized proximal-distal ureter contraction (Figure 3, D and E, and Supplemental Video 8). Consistent with normal ureter peristalsis, we observed restoration of Kit and Hcn3 expression in the renal pelvis and proximal ureter (Figure 3, F and $\mathrm{G})$. Together, these results indicated that increased GLI3 repressor relative to GLI activator results in decreased Kit and Hcn3 expression, abnormal ureter peristalsis, functional obstruction, hydroureter, and hydronephrosis.

\section{Discussion}

The underlying mechanisms that regulate normal ureter function and contribute to congenital functional obstructions are poorly understood. Mutations in genes encoding Hh signaling effectors in humans and mice with hydroureter have implicated Hh-dependent signaling in ureter development and function. Here, we identified what we believe to be a novel function for Hh signaling in the 
regulation of 2 distinct cell populations required for the initiation and transmission of coordinated ureter peristalsis. Targeted inactivation of Smo in the mesenchyme surrounding the renal pelvis and upper ureter resulted in nonobstructive hydronephrosis and hydroureter characterized by abnormal ureter peristalsis. Smo-deficient mice exhibited reduced expression of Kit and Hcn3, markers of 2 distinct cell populations required for coordinated unidirectional ureter peristalsis. A similar phenotype in a mouse model of PHS suggested a pathogenic role for GLI3 repressor in Smo-deficient embryos. Indeed, inactivation of Gli3 in the Smo-deficient background normalized renal phenotype, ureter function, and Kit and Hcn 3 expression.

Functional urinary tract obstruction in the absence of structural abnormalities of the ureter. Structural abnormalities of the ureter - including delayed/improper differentiation and maturation of the smooth muscle $(8,19-24)$, defects in the ureteral stromal layers (22), and urothelium abnormalities (30) - are common causes of congenital murine urinary tract dilation. Abnormalities associated with the smooth muscle layer have been strongly implicated in functional obstruction (20-22). The Smo-deficient mice described herein are a model of functional obstruction in the absence of ureter structural abnormalities. This abnormality is similar in type to $50 \%$ of all human congenital urinary tract dilatations. Interestingly, inactivation of $S h b$ targeted to the ureteric epithelium results in delayed smooth muscle cell differentiation and severe hydroureter (8). We performed careful histological and immunohistochemical analyses of the smooth muscle layer in Smo-deficient mice and could not detect any abnormalities in onset and progression of differentiation, organization of smooth muscle cells and layers, and smooth muscle thickness. This is likely due to the fact that Smo inactivation in the ureter mesenchyme under the control of Rarb2-Cre occurs after the critical stage for ureter smooth muscle development. Despite the absence of morphological abnormalities in Smo-deficient ureter smooth muscle, we have not yet determined whether smooth muscle gap junctions, which are required to facilitate the spread of action potentials from cell to cell during ureter peristalsis, are maintained. However, the abnormalities associated with the Kit and Hon 3 pacemaker cell populations strongly suggest that the primary pathogenic mechanism involves the initiation and primary transmission of the peristaltic signal in the renal pelvis and proximal ureter.

Kit and Hcn 3 in functional ureter obstruction. Our data demonstrated that Hh signaling regulates ureter peristalsis via control of Kit and Hcn 3 expression. Reduced Kit expression in Smo-deficient mice is consistent with published results implicating Kit in peristaltic function. Inhibition of Kit in isolated ureter cultures using neutralizing antibodies results in inhibition of unidirectional and coordinated peristalsis and fibrillation-like movements of the proximal ureter, but has no effect on the differentiation of the ureter cell layers (11). Decreased or absent Kit immunoreactivity in the gut has been detected in Hirschsprung disease and chronic intestinal pseudo-obstruction caused by abnormal gastrointestinal peristalsis $(31,32)$. Finally, white spotting mice, characterized by inactivating mutations in Kit, exhibit uncoordinated intestinal peristalsis (33). Although early kidney development is unperturbed in these mice, to our knowledge, their functional development of the ureter has not been defined. Likewise, the importance of $\mathrm{Hcn} 3$ in ureter contraction initiation is supported by the observation that inhibition of $\mathrm{Hcn} 3$ using a $\mathrm{HCN}$ ion channel inhibitor in urinary tract cultures causes abnormal peristalsis (14). Interestingly, Kit and
Hcn 3 expression in control urinary tracts did not overlap (data not shown), which suggests that Kit and Hcn3 mark distinct cell populations. It is unclear whether these distinct cell populations work independently or in unison. Since inhibition of either Kit or $\mathrm{Hcn} 3$ resulted in severe peristaltic defects, it is clear that both are essential for ureter function.

$\mathrm{Hb}$-dependent control of Kit and Hcn3. In silico analysis of the Kit promoter region demonstrated 2 GLI consensus-binding sites within $1 \mathrm{~kb}$ of the ATG translational start site (data not shown). Furthermore, genomic DNA encoding Kit is bound by GLI3 repressor in the mammalian limb bud (34). These observations suggest that GLI3 repressor directly inhibits Kit transcription. In contrast, GLI consensus-binding sites have not been identified in the promoter region of Hcn3. Further investigation into the origin of both the Kit and Hcn3 cell populations will be informative in establishing whether GLI3 repressor disrupts the differentiation, survival, and/or migration of these cell populations or whether GLI3 repressor represses Kit or Hcn 3 expression in their respective differentiated cells. Currently, Kit and Hcn3 are the sole markers for their distinct cell populations. The identification of other specific markers is required to elucidate the precise effects of Hhdependent signaling on these cell populations. In the gastrointestinal tract, Kit-positive interstitial cells of Cajal are believed to originate from the gut mesenchyme $(35,36)$. The precise origin of Kit-positive ICC-LCs in the urinary system is unknown. Lineage tracing will be useful in identifying the origins of both the Kit and the Hon 3 cell populations.

A bifunctional role of Hh signaling during establishment of ureter peristalsis. Hh signaling has been previously implicated in ureteral smooth muscle development $(5,7,8,37,38)$. Here, using application of Rarb2-Cre to analyze Hh signaling in the ureter mesenchyme, we have identified what we believe to be a novel additional requirement for $\mathrm{Hh}$ signaling in unidirectional and coordinated ureter contractions via regulation of 2 distinct cell populations believed to be important in electrical activity initiation and transmission. We have previously shown that Hh signaling activity is specifically localized to the mesenchyme surrounding the ureter (4). Here, we propose a model whereby Hh signaling activity is required during early patterning of the ureter mesenchyme for timely and proper differentiation of the ureter cell layers, specifically the ureter smooth muscle. In addition, we propose that $\mathrm{Hh}$ signaling activity is also required during the later stages of ureter morphogenesis for the establishment or maintenance of specific cell populations necessary for establishment of unidirectional and coordinated peristalsis (Figure $3 \mathrm{H}$ ). Interestingly, it seems likely that the primary role for Hh signaling activity in the ureter mesenchyme is to prevent the deleterious actions of GLI3 repressor rather than to directly promote ureter morphogenesis itself. To our knowledge, this is the first study to explore the pathogenic mechanisms controlling hydroureter in the absence of structural abnormalities. Furthermore, these results implicate misregulation of Hh signaling in defective peristalsis and in the pathogenesis of human hydroureter and hydronephrosis.

\section{Methods}

Mice. Rarb2-Cre mice (provided by R. Behringer, University of Texas, Houston, Texas, USA; ref. 18) were mated with $\mathrm{Smo}^{+/-}$mice (17) to generate Rarb2-Cre; $\mathrm{Smo}^{+/-}$males. These males were mated to homozygous Smo conditional (Smo ${ }^{\text {loxP/loxP }}$; ref. 39) females (provided by C.C. Hui, Hospital for Sick Children) to generate Rarb2-Cre;Smo ${ }^{l o x P /-}$ progeny in which Smo is specifically 
deleted from the intermediate mesoderm. For rescue experiments, Gli3 ${ }^{X t /+}$ mice (provided by C.C. Hui; ref. 40) were mated to Rarb2-Cre;Smo ${ }^{+/-}$and Smo loxP/loxP mice to ultimately generate Rarb2-Cre; $\mathrm{Smo}^{+/-;}$;li3 ${ }^{\mathrm{Xt} / /+}$ male and $S m o^{\text {loxP } / \text { loxP }} ; \mathrm{Gli3}^{\mathrm{XtJ} / \mathrm{+}}$ female progeny. These mice were intercrossed to generate Rarb2-Cre;Smo ${ }^{\text {loxP/-; }}$ Gli3 ${ }^{X t / X t]}$ embryos. Gli3 ${ }^{\Delta 699 /+}$ heterozygous mice (10) were intercrossed to generate Gli3 ${ }^{\Delta 699 / \Delta 699}$ embryos. Gli2 $2^{z d /+}$ heterozygous mice (provided by C.C. Hui; ref. 9) were intercrossed to generate Gli2 zfd/zfd embryos. PCR genotyping for each allele was performed as previously described $(9,10,17,18,39)$. Gli3 ${ }^{X t}$ heterozygote and homozygote mice were genotyped according to their characteristic limb phenotypes. For analysis of Rarb2-Cre expression, Rarb2-Cre mice were mated with Gt(ROSA)26Sor(ROSA) mice (41). Littermates were used for all experiments in which normal and mutant embryos were compared. Experiments using mice were approved in advance by the Animal Ethics Committee at Hospital for Sick Children and were carried out in accordance with the Canadian Council of Animal Care.

Histological analyses. Paraffin-embedded kidney sections were analyzed by histology after generating $4-\mu \mathrm{m}$ tissue sections and staining with hematoxylin and eosin or with Masson trichrome. Immunofluorescence was performed on formalin-fixed, paraffin-embedded kidney sections or $4 \%$ PFA-fixed, OCT-embedded frozen sections using anti- $\alpha$-SMA (SigmaAldrich, 1:500 dilution), anti-UpkIII (Progen Biotechnik, 1:10 dilution), anti-pan-cytokeratin (Sigma-Aldrich, 1:100 dilution), anti-CD117 (Kit; DAKO, 1:50 dilution), Hcn3 (Millipore, 1:500 dilution). Alexa Fluor 488 and Alexa Fluor 568 goat anti-mouse, goat anti-rat, or goat anti-rabbit (Invitrogen, 1:1,000 dilution) were used as secondary antibodies. Whole mount immunofluorescence was performed as described previously (42) with anti-Calbindin- $\mathrm{D}_{28 \mathrm{~K}}$ (Sigma-Aldrich, 1:200 dilution) and secondary Alexa Fluor 488 goat anti-mouse (Invitrogen, 1:100 dilution). $\beta$-Galactosidase staining was performed as previously described (4).

Intrapelvic dye injections. Visualization of the ureteropelvic lumen was performed as previously described (19). Briefly, bromo-phenol blue dye was injected into the renal pelvis of isolated whole urogenital systems at E18.5 using a pulled-out Pasteur glass pipette.
Metanephric mesenchyme isolation and real-time RT-PCR. Tissue isolations and real-time RT-PCR were performed as previously described (4).

Embryonic ureter cultures. E15.5 ureters were dissected and cultured for 5 days on $0.4-\mu \mathrm{m}$ membranes (Falcon) in wells containing DMEM/HamsF12 culture media (Gibco, Invitrogen) supplemented with transferrin $(5 \mu \mathrm{g} / \mathrm{ml})$, penicillin $(100 \mu \mathrm{g} / \mathrm{ml})$, and streptomycin $(100 \mathrm{U} / \mathrm{ml})$. Time-lapse imaging of ureters was performed on a Zeiss AxioVert 200M microscope. Images were captured every $500 \mathrm{~ms}$ for a total duration of 90 seconds. Peristalsis of ureters was quantified by measuring the lumen diameter at fixed points throughout the contraction period using Axiovision Release software (version 4.6.3.0).

In situ bybridization. In situ hybridization was performed on paraffinembedded sections ( $4 \mu \mathrm{m})$ using DIG-labeled cDNA probes encoding Ret, $W n t 11, W n t 7 b$, and $W n t 9 b$ as previously described (4).

Statistics. Statistical analysis was performed using GraphPad Prism software (version 5.0c). Data were analyzed using 2-tailed Student's $t$ test. A probability of less than 0.05 was considered to indicate statistical significance. Values are given as mean \pm SEM.

\section{Acknowledgments}

We thank Ulrich Ruther (University of Dusseldorf, Dusseldorf, Germany) for permission to use the Gli3 ${ }^{\Delta 699 /+}$ mice. This research was supported by operating grants from the Canadian Institutes of Health Research and the Kidney Foundation of Canada and by a Canada Research Chair (to N.D. Rosenblum).

Received for publication October 22, 2010, and accepted in revised form December 15, 2010.

Address correspondence to: Norman D. Rosenblum, Division of Nephrology, Hospital for Sick Children, 555 University Avenue, Toronto, Ontario, Canada M5G 1X8. Phone: 416.813.5667; Fax: 416.813.6271; E-mail: norman.rosenblum@sickkids.ca.
1. Dudley JA, Haworth JM, McGraw ME, Frank JD, Tizard EJ. Clinical relevance and implications of antenatal hydronephrosis. Arch Dis Child Fetal Neonatal Ed. 1997;76(1):F31-34.

2. Johnson CE, Elder JS, Judge NE, Adeeb FN, Grisoni ER, Fattlar DC. The accuracy of antenatal ultrasonography in identifying renal abnormalities. Am J Dis Child. 1992;146(10):1181-1184.

3. Chevalier RL. Pathophysiology of obstructive nephropathy in the newborn. Semin Nephrol. 1998; 18(6):585-593.

4. Cain JE, et al. GLI3 repressor controls nephron number via regulation of $\mathrm{Wnt} 11$ and Ret in ureteric tip cells. PLoS One. 2009;4(10):e7313.

5 . Hall JG, et al. Congenital hypothalamic hamartoblastoma, hypopituitarism, imperforate anus and postaxial polydactyly--a new syndrome? Part I: clinical, causal, and pathogenetic considerations. Am J Med Genet. 1980;7(1):47-74.

6. $\mathrm{Hu} \mathrm{MC}$, et al. GLI3-dependent transcriptional repression of Gli1, Gli2 and kidney patterning genes disrupts renal morphogenesis. Development. 2006; 133(3):569-578.

7. Johnston JJ, et al. Molecular and clinical analyses of Greig cephalopolysyndactyly and Pallister-Hall syndromes: robust phenotype prediction from the type and position of GLI3 mutations. Am J Hum Genet. 2005;76(4):609-622.

8. Yu J, Carroll TJ, McMahon AP. Sonic hedgehog regulates proliferation and differentiation of mesenchymal cells in the mouse metanephric kidney. Development. 2002;129(22):5301-5312.

9. Mo R, et al. Specific and redundant functions of Gli2 and Gli3 zinc finger genes in skeletal pat- terning and development. Development. 1997; 124(1):113-123.

10. Bose J, Grotewold L, Ruther U. Pallister-Hall syndrome phenotype in mice mutant for Gli3. Hum Mol Genet. 2002;11(9):1129-1135.

11. David SG, Cebrian C, Vaughan ED Jr, Herzlinger D. c-kit and ureteral peristalsis. J Urol. 2005;173(1):292-295.

12. Hashitani H, Lang RJ. Functions of ICC-like cells in the urinary tract and male genital organs. J Cell Mol Med. 2010;14(6A):1199-1211.

13. Lang RJ, Tonta MA, Zoltkowski BZ, Meeker WF, Wendt I, Parkington HC. Pyeloureteric peristalsis: role of atypical smooth muscle cells and interstitial cells of Cajal-like cells as pacemakers. J Physiol. 2006;576(pt 3):695-705.

14. Hurtado R, Bub G, Herzlinger D. The pelvis-kidney junction contains $\mathrm{HCN} 3$, a hyperpolarization-activated cation channel that triggers ureter peristalsis. Kidney Int. 2010;77(6):500-508.

15. Ishii TM, Takano M, Xie LH, Noma A, Ohmori H. Molecular characterization of the hyperpolarization-activated cation channel in rabbit heart sinoatrial node. J Biol Chem. 1999;274(18):12835-12839.

16. Ludwig A, Zong X, Stieber J, Hullin R, Hofmann F, Biel M. Two pacemaker channels from human heart with profoundly different activation kinetics. EMBO J. 1999;18(9):2323-2329.

17. Zhang XM, Ramalho-Santos M, McMahon AP. Smoothened mutants reveal redundant roles for Shh and Ihh signaling including regulation of L/R symmetry by the mouse node. Cell. 2001; 106(2):781-792.

18. Kobayashi A, Kwan KM, Carroll TJ, McMahon
AP, Mendelsohn CL, Behringer RR. Distinct and sequential tissue-specific activities of the LIMclass homeobox gene Lim1 for tubular morphogenesis during kidney development. Development. 2005;132(12):2809-2823.

19. Airik R, Bussen M, Singh MK, Petry M, Kispert A. Tbx18 regulates the development of the ureteral mesenchyme. J Clin Invest. 2006;116(3):663-674.

20. Caubit $X$, et al. Teashirt 3 is necessary for ureteral smooth muscle differentiation downstream of $\mathrm{SHH}$ and BMP4. Development. 2008;135(19):3301-3310.

21. Chang $\mathrm{CP}$, et al. Calcineurin is required in urinary tract mesenchyme for the development of the pyeloureteral peristaltic machinery. J Clin Invest. 2004; 113(7):1051-1058.

22. Mahoney ZX, et al. Discs-large homolog 1 regulates smooth muscle orientation in the mouse ureter. Proc Natl Acad Sci U S A. 2006;103(52):19872-19877.

23. Miyazaki Y, Oshima K, Fogo A, Ichikawa I. Evidence that bone morphogenetic protein 4 has multiple biological functions during kidney and urinary tract development. Kidney Int. 2003;63(3):835-844.

24. Miyazaki Y, et al. Angiotensin induces the urinary peristaltic machinery during the perinatal period. J Clin Invest. 1998;102(8):1489-1497.

25. Cano-Gauci DF, et al. Glypican-3-deficient mice exhibit developmental overgrowth and some of the abnormalities typical of Simpson-Golabi-Behmel syndrome. J Cell Biol. 1999;146(1):255-264.

26. Hartwig S, Bridgewater D, Di Giovanni V, Cain J, Mishina Y, Rosenblum ND. BMP receptor ALK3 controls collecting system development. J Am Soc Nephrol. 2008;19(1):117-124

27. Yu J, Carroll TJ, Rajagopal J, Kobayashi A, Ren Q, 
McMahon AP. A Wnt7b-dependent pathway regulates the orientation of epithelial cell division and establishes the cortico-medullary axis of the mammalian kidney. Development. 2009;136(1):161-171.

28. Metzger R, Neugebauer A, Rolle U, Bohlig L, Till H. C-Kit receptor (CD117) in the porcine urinary tract. Pediatr Surg Int. 2008;24(1):67-76.

29. Pezzone MA, et al. Identification of c-kit-positive cells in the mouse ureter: the interstitial cells of Cajal of the urinary tract. Am J Physiol Renal Physiol. 2003;284(5):F925-F929.

30. Hu P, et al. Ablation of uroplakin III gene results in small urothelial plaques, urothelial leakage, and vesicoureteral reflux. J Cell Biol. 2000;151(5):961-972.

31. Rolle U, Yoneda A, Solari V, Nemeth L, Puri P. Abnormalities of C-Kit-positive cellular network in isolated hypoganglionosis. J Pediatr Surg. 2002; 37(5):709-714.

32. Streutker CJ, Huizinga JD, Campbell F, Ho J, Riddell RH. Loss of CD117 (c-kit)- and CD34-positive ICC and associated CD34-positive fibroblasts defines a subpopulation of chronic intestinal pseudoobstruction. Am J Surg Pathol. 2003;27(2):228-235.

33. Nocka K, et al. Molecular bases of dominant negative and loss of function mutations at the murine c-kit/white spotting locus: W37, Wv, W41 and W. EMBO J. 1990;9(6):1805-1813.

34. Vokes SA, Ji H, Wong WH, McMahon AP. A genomescale analysis of the cis-regulatory circuitry underlying sonic hedgehog-mediated patterning of the mammalian limb. Genes Dev. 2008;22(19):2651-2663.

35. Lecoin L, Gabella G, Le Douarin N. Origin of the c-kit-positive interstitial cells in the avian bowel. Development. 1996;122(3):725-733

36. Young HM, Ciampoli D, Southwell BR, Newgreen DF. Origin of interstitial cells of Cajal in the mouse intestine. Dev Biol. 1996;180(1):97-107.

37. Pallister PD, Hecht F, Herrman J. Three additional cases of the congenital hypothalamic "hamartoblastoma" (Pallister-Hall) syndrome. Am J Med
Genet. 1989;33(4):500-501.

38. Sama A, Mason JD, Gibbin KP, Young ID, Hewitt M. The Pallister-Hall syndrome. J Med Genet. 1994; 31(9):740.

39. Long F, Zhang XM, Karp S, Yang Y, McMahon AP. Genetic manipulation of hedgehog signaling in the endochondral skeleton reveals a direct role in the regulation of chondrocyte proliferation. Development. 2001;128(24):5099-5108.

40. Hui CC, Joyner AL. A mouse model of greig cephalopolysyndactyly syndrome: the extra-toesJ mutation contains an intragenic deletion of the Gli3 gene. Nat Genet. 1993;3(3):241-246.

41. Soriano P. Generalized lacZ expression with the ROSA26 Cre reporter strain. Nat Genet. 1999; 21(1):70-71.

42. Cain JE, Nion T, Jeulin D, Bertram JF. Exogenous BMP-4 amplifies asymmetric ureteric branching in the developing mouse kidney in vitro. Kidney Int. 2005;67(2):420-431. 